\title{
Derivative of Eigensolutions of Nonviscously Damped Linear Systems
}

\author{
Sondipon Adhikari* \\ University of Cambridge, Cambridge, England CB2 1PZ, United Kingdom
}

\begin{abstract}
Derivatives of eigenvalues and eigenvectors of multiple-degree-of-freedom damped linear dynamic systems with respect to arbitrary design parameters are presented. In contrast to the traditional viscous damping model, a more general nonviscous damping model is considered. The nonviscous damping model is such that the damping forces depend on the past history of velocities via convolution integrals over some kernel functions. Because of the general nature of the damping, eigensolutions are generally complex valued, and eigenvectors do not satisfy any orthogonality relationship. It is shown that under such general conditions the derivative of eigensolutions can be expressed in a way similar to that of undamped or viscously damped systems. Numerical examples are provided to illustrate the derived results.
\end{abstract}

\begin{tabular}{|c|c|}
\hline & Nomenclature \\
\hline$C$ & $=$ viscous damping matrix \\
\hline $\mathbb{C}$ & space of complex numbers \\
\hline$c$ & damping constant \\
\hline $\boldsymbol{D}(s)$ & dynamic stiffness matrix \\
\hline $\boldsymbol{G}(s)$ & $=$ damping function in the Laplace domain \\
\hline$G(t)$ & $=$ damping function in the time domain \\
\hline $\boldsymbol{I}$ & $=$ identity matrix \\
\hline$\Im()$ & $=$ imaginary part of () \\
\hline$i$ & $=$ unit imaginary number, $\sqrt{ }-1$ \\
\hline $\boldsymbol{K}$ & $=$ stiffness matrix \\
\hline$k_{1}, k_{2}, k_{3}$ & $=$ spring constants \\
\hline$M$ & mass matrix \\
\hline$m$ & order of the characteristic polynomial \\
\hline$N$ & degrees of freedom of the system \\
\hline $\boldsymbol{O}$ & $=$ null matrix \\
\hline$p$ & $=$ design parameter \\
\hline $\mathbb{R}$ & $=$ space of real numbers \\
\hline$s$ & $=$ Laplace domain parameter \\
\hline$t$ & $=$ time \\
\hline $\boldsymbol{u}_{j}$ & $j$ th eigenvector of the system \\
\hline$\overline{\boldsymbol{u}}(s)$ & Laplace transform of $\boldsymbol{u}(t)$ \\
\hline $\boldsymbol{u}(t)$ & response vector \\
\hline$\delta_{j k}$ & $=$ Kroneker delta function \\
\hline$\delta(t)$ & $=$ Dirac delta function \\
\hline$\theta_{j}$ & $=$ normalization constant for $j$ th mode \\
\hline$\lambda_{j}$ & $=j$ th eigenvalue of the system \\
\hline$\mu_{1}, \mu_{2}$ & $\begin{aligned}= & \text { parameters of the Golla-Hughes-McTavish } \\
& \text { (see Refs. } 23 \text { and 32) damping model }\end{aligned}$ \\
\hline$\nu(s)$ & $=$ diagonal matrix containing $v_{j}(s)$ \\
\hline$v_{j}(s)$ & $=j$ th eigenvalue of $\boldsymbol{D}(s)$ \\
\hline$\Phi(s)$ & $=$ matrix containing $\phi_{j}(s)$ \\
\hline$\phi_{j}(s)$ & $=j$ th eigenvector of $\boldsymbol{D}(s)$ \\
\hline$\omega_{j}$ & $=j$ th undamped natural frequency \\
\hline$|()|$ & $=l_{2}$ norm of the vector () \\
\hline
\end{tabular}

Subscripts
$=$ elastic modes

$=$ nonviscous modes
Superscripts

$\begin{array}{ll}T & =\text { matrix transpose } \\ -1 & =\text { matrix inverse } \\ \cdot & =\text { derivative with respect to } t \\ * & =\text { complex conjugate }\end{array}$

\section{Introduction}

D URING the design process of a structure, it is often required to make changes in the design parameters so that the design is optimal. When dynamic problems are considered, the interest of designers lies in understanding the changes in natural frequencies and mode shapes due to the changes in the system parameters. The sensitivity of eigensolutions, or more precisely, the derivative of eigensolutions with respect to the design parameters has an important role in such studies because it helps to avoid repeated calculations. Also the eigensensitivity analysis plays a major role in the system identification problems and in the analysis of stochastically perturbed dynamic systems. Because of such widespread applications, the calculation of derivative of eigenvalues and eigenvectors has emerged an important area of research over past four decades.

In one of the earliest works, Fox and Kapoor ${ }^{1}$ gave exact expressions for the derivative of eigenvalues and eigenvectors with respect to any design variable. Their results were obtained in terms of changes in the system property matrices and the eigensolutions of the structure and have been used extensively in a wide range of application areas of structural dynamics. The expressions derived in Ref. 1 are valid for symmetric undamped systems. Later, many authors ${ }^{2-5}$ extended Fox and Kapoor's ${ }^{1}$ approach to determine eigensolution derivatives for more general asymmetric systems. For these kinds of systems, Nelson ${ }^{6}$ proposed an efficient method to calculate the derivative of eigenvectors that requires only the eigenvalue and eigenvector under consideration. A review on calculating the derivatives of eigenvalues and eigenvectors associated with general (non-Hermitian) matrices may be found by Murthy and Haftka. ${ }^{7}$

The cited works do not explicitly consider the damping present in the system. To apply these results to systems with general nonproportional (viscous) damping, it is required to convert the equations of motion into state-space form (for example, see Ref. 8). Although exact in nature, state-space methods require significant numerical effort as the size of the problem doubles. Moreover, these methods also lack some of the intuitive simplicity of the analysis based on $N$ space. For these reasons some authors have considered the problem of the calculation of derivatives of eigensolutions of viscously damped systems in $N$ space. One of the earliest work to consider damping was by Cardani and Mantegazza ${ }^{9}$ in the context of flutter problems. Note that, unlike undamped systems, in damped systems the eigenvalues and eigenvectors, and consequently their derivatives, become complex in general. Adhikari ${ }^{10}$ derived an exact expression for the derivative of complex eigenvalues and eigenvectors 
The results were expressed in terms of the complex eigenvalues and eigenvectors of the second-order system and the state-space representation of the equation of motion was avoided. Lee et al. ${ }^{1,12}$ have proposed an approach to determine natural frequency and mode shape sensitivities of damped systems. However, unlike Adhikari, ${ }^{10}$ who employed a complex modal expansion approach, their method for the calculation of eigenvector derivative involves direct matrix inversion. Adhikari ${ }^{13}$ suggested an approximate method to calculate the derivative of complex modes using a modal series involving only classical normal modes. Later, Friswell and Adhikari ${ }^{14}$ extended Nelson's method ${ }^{6}$ to nonproportionally damped systems with complex modes. Recently, Adhikari and Friswell ${ }^{15}$ have derived the first and second-order derivative of complex eigensolutions of more general asymmetric nonconservative systems.

The studies so far consider only viscous damping model. However, it is well known that viscous damping is not the only damping model within the scope of linear analysis, examples are damping in composite materials, ${ }^{16}$ energy dissipation in structural joints, ${ }^{17,18}$ and damping mechanism in composite beams, ${ }^{19}$ to mention a few. We consider a class of nonviscous damping models in which the damping forces depend on the past history of motion via convolution integrals over some kernel functions. The equations of motion describing free vibration of an $N$-degree-of-freedom linear system with such damping can be expressed by

$$
\boldsymbol{M} \ddot{\boldsymbol{u}}(t)+\int_{-\infty}^{t} \boldsymbol{G}(t-\tau) \dot{\boldsymbol{u}}(\tau) \mathrm{d} \tau+\boldsymbol{K} \boldsymbol{u}(t)=\mathbf{0}
$$

where $\boldsymbol{M}$ and $\boldsymbol{K} \in \mathbb{R}^{N \times N}$ are the mass and stiffness matrices, $G(t) \in \mathbb{R}^{N \times N}$ is the matrix of kernel functions, and $\mathbf{0}$ is an $N \times 1$ vector of zeros. In the special case when $G(t-\tau)=\boldsymbol{C} \delta(t-\tau)$, Eq. (1) reduces to the case of viscously damped systems. The damping model of this kind is a further generalization of the familiar viscous damping. Recently, Adhikari and Woodhous e ${ }^{20}$ proposed a method for identification of exponential nonviscous damping model where the kernel function matrix has the special form $G(t-\tau)=\mu \boldsymbol{C} e^{-\mu(t-\tau)}$.

The central aim of this paper is to extend the eigensensitivity analysis to nonviscously damped systems of the form (1). In the next section, we briefly discuss eigenvalues and eigenvectors of such systems. In the two subsequent sections, the derivative of eigenvalues and eigenvectors are derived. Note that, unlike viscously damped systems, the conversion of Eq. (1) into state-space form does not give any advantage because the eigenvalue problem in state space cannot be cast in the form of the conventional matrix eigenvalue problem involving constant matrices. For this reason, the approach adopted here does not employ the state-space formulation of the equations of motion. An application of the derived expressions for the derivative of eigensolutions is illustrated by considering a twodegree-of-freedom system with nonviscous damping.

\section{Eigenvalues and Eigenvectors}

The determination of eigenvalues and eigenvectors of nonviscously damped systems has been recently discussed by Adhikari. ${ }^{21}$ Here we briefly outline the topics required for further development. Taking the Laplace transform of Eq. (1), we have

$s^{2} \boldsymbol{M} \overline{\boldsymbol{u}}(s)+s \boldsymbol{G}(s) \overline{\boldsymbol{u}}(s)+\boldsymbol{K} \overline{\boldsymbol{u}}(s)=\mathbf{0} \quad$ or $\quad \boldsymbol{D}(s) \overline{\boldsymbol{u}}(s)=\mathbf{0}$

where the dynamic stiffness matrix

$$
\boldsymbol{D}(s)=s^{2} \boldsymbol{M}+s \boldsymbol{G}(s)+\boldsymbol{K} \in \mathbb{C}^{N \times N}
$$

and where $\overline{\boldsymbol{u}}(s)=\mathcal{L}[\boldsymbol{u}(t)] \in \mathbb{C}^{N}, \boldsymbol{G}(s)=\mathcal{L}[\boldsymbol{G}(t)] \in \mathbb{C}^{N \times N}$, and $\mathcal{L}[]$ is the Laplace transform. In the context of structural dynamics, $s=i \omega$, where $\omega \in \mathbb{R}^{+}$is the frequency. We consider the damping to be nonproportional. (Conditions for proportionality of nonviscous damping were derived in Ref. 22.) That is, the mass and stiffness matrices as well as the matrix of kernel functions cannot be simultaneously diagonalized by any linear transformation. However, it is assumed that $\boldsymbol{M}^{-1}$ exist and $\boldsymbol{G}(s)$ is such that the motion is dissipative. The conditions that $\boldsymbol{G}(s)$ must satisfy to produce dissipative motion were given by Golla and Hughes. ${ }^{23}$
The eigenvalue problem associated with Eq. (1) can be defined from Eq. (2) as

$$
\left[\lambda_{j}^{2} \boldsymbol{M}+\lambda_{j} \boldsymbol{G}\left(\lambda_{j}\right)+\boldsymbol{K}\right] \boldsymbol{u}_{j}=\mathbf{0} \quad \text { or } \quad \boldsymbol{D}\left(\lambda_{j}\right) \boldsymbol{u}_{j}=\mathbf{0}
$$

where $\boldsymbol{u}_{j} \in \mathbb{C}^{N}$ is the $j$ th eigenvector. The eigenvalues $\lambda_{j}$ are roots of the characteristic equation

$$
\operatorname{det}\left[s^{2} \boldsymbol{M}+s \boldsymbol{G}(s)+\boldsymbol{K}\right]=0
$$

For the linear viscoelastic case, it can be shown that, ${ }^{24,25}$ in general, the elements of $\boldsymbol{G}(s)$ can be represented by

$$
G_{j k}(s)=\frac{p_{j k}(s)}{q_{j k}(s)}
$$

where $p_{j k}(s)$ and $q_{j k}(s)$ are finite-order polynomials in $s$ and the degree of $p_{j k}(s)$ is not more than that of $q_{j k}(s)$. Under such assumptions, in general, the order of the characteristic equation $m$ is more than $2 N$, that is, $m=2 N+p ; p \geq 0$. Thus, although the system has $N$ degrees of freedom, the number of eigenvalues is more than $2 N$. This is a major difference between nonviscously damped systems and viscously damped systems where the number of eigenvalues is exactly $2 N$, including any multiplicities. Following Adhikari, ${ }^{21}$ one may group the eigenvectors as 1) elastic modes (corresponding to $N$ complex conjugate pairs of eigenvalues) and 2) nonviscous modes (corresponding to the additional $p$ eigenvalues). The elastic modes are related to the $N$ modes of vibration of structural systems. In this paper we assume that all $m$ eigenvalues are distinct.

Adhikari ${ }^{26}$ discussed the orthogonality and the normalization relationships of the eigenvectors. Noting the symmetry of $\boldsymbol{D}(s)$ and using Eq. (4) for the $k$ th set, we can obtain

$$
\boldsymbol{u}_{j}^{T}\left[\boldsymbol{D}\left(\lambda_{k}\right)-\boldsymbol{D}\left(\lambda_{j}\right)\right] \boldsymbol{u}_{k}=0
$$

Because $\lambda_{j}$ and $\lambda_{k}$ are assumed to be distinct for different $j$ and $k$, Eq. (7) can be divided by $\left(\lambda_{k}-\lambda_{j}\right)$ to obtain

$$
\boldsymbol{u}_{j}^{T}\left[\frac{\boldsymbol{D}\left(\lambda_{k}\right)-\boldsymbol{D}\left(\lambda_{j}\right)}{\lambda_{k}-\lambda_{j}}\right] \boldsymbol{u}_{k}=0, \quad \forall j, k ; \quad j \neq k
$$

This equation may be regarded as the orthogonality relationship of the eigenvectors. It is easy to verify that, in the undamped limit, Eq. (8) degenerates to the familiar mass orthogonality relationship of the undamped eigenvectors. Assume $\delta_{\lambda}=\lambda_{k}-\lambda_{j}$, and rewrite Eq. (8) as

$$
\boldsymbol{u}_{j}^{T}\left[\frac{\boldsymbol{D}\left(\lambda_{j}+\delta_{\lambda}\right)-\boldsymbol{D}\left(\lambda_{j}\right)}{\delta_{\lambda}}\right] \boldsymbol{u}_{j}=0
$$

Consider the case when $\lambda_{k} \rightarrow \lambda_{j}$, that is, $\delta_{\lambda} \rightarrow 0$. When $\lambda_{k}=\lambda_{j}$ is substituted in Eq. (7), it is easy to verify that when the right-hand side is zero the relationship represents a trivial case. For the nontrivial case, the right-hand side of Eq. (7) must be nonzero as $\lambda_{k} \rightarrow \lambda_{j}$. Thus, for $\delta_{\lambda} \rightarrow 0$, Eq. (9) reads

$$
\begin{gathered}
\left.\boldsymbol{u}_{j}^{T} \frac{\partial \boldsymbol{D}(s)}{\partial s}\right|_{s=\lambda_{j}} \boldsymbol{u}_{j}=\theta_{j} \quad \text { or } \\
\boldsymbol{u}_{j}^{T}\left[2 \lambda_{j} \boldsymbol{M}+\boldsymbol{G}\left(\lambda_{j}\right)+\left.\lambda_{j} \frac{\partial \boldsymbol{G}(s)}{\partial s}\right|_{s=\lambda_{j}}\right] \boldsymbol{u}_{j}=\theta_{j} \\
\forall j=1, \ldots, m
\end{gathered}
$$

where $\theta_{j} \in \mathbb{C}$ is some nonzero constant. Note that Eq. (10) reduces to the corresponding normalization relationship for viscously damped systems (for example, see Refs. 27 and 28), when $\boldsymbol{G}(s)$ is constant with respect to $s$. Numerical values of $\theta_{j}$ can be selected in various ways. For example, one can choose $\theta_{j}=2 \lambda_{j}, \forall j$, which reduces to $\boldsymbol{u}_{j}^{T} \boldsymbol{M} \boldsymbol{u}_{j}=1$ when the damping is zero. This is consistent with the familiar unity modal mass convention. One may also choose $\theta_{j}=1, \forall j$. Theoretical analysis becomes easiest with this normalization. However, as pointed out by Fawzy ${ }^{29}$ and Vigneron, ${ }^{27}$ 
in the context of viscously damped systems, this normalization is inconsistent with undamped or classically damped modal theories. Alternatively, one may choose other kind of normalizations, for example, $\max \left(\boldsymbol{u}_{j}\right)=1$ or $\left|\boldsymbol{u}_{j}\right|=1$. In such cases, one simply calculates the numerical values of $\theta_{j}$ from Eq. (10) and uses them subsequently. Further discussions on the normalization of complex modes may be found in Refs. 26 and 30.

\section{Derivative of Eigenvalues}

Suppose that the system matrices in Eq. (1) are functions of some design parameter $p$. In this section, we intend to obtain an expression of the derivative of the $j$ th eigenvalue with respect to the design parameter $p$. Differentiating Eq. (4) with respect to $p$, one obtains

$$
\begin{gathered}
{\left[2 \lambda_{j} \frac{\partial \lambda_{j}}{\partial p} \boldsymbol{M}+\lambda_{j}^{2} \frac{\partial \boldsymbol{M}}{\partial p}+\frac{\partial \lambda_{j}}{\partial p} \boldsymbol{G}\left(\lambda_{j}\right)+\lambda_{j} \frac{\partial\left[\boldsymbol{G}\left(\lambda_{j}\right)\right]}{\partial p}+\frac{\partial \boldsymbol{K}}{\partial p}\right] \boldsymbol{u}_{j}} \\
+\left[\lambda_{j}^{2} \boldsymbol{M}+\lambda_{j} \boldsymbol{G}\left(\lambda_{j}\right)+\boldsymbol{K}\right] \frac{\partial \boldsymbol{u}_{j}}{\partial p}=\mathbf{0}
\end{gathered}
$$

The term $\partial\left[\boldsymbol{G}\left(\boldsymbol{\lambda}_{j}\right)\right] / \partial p$ appearing in Eq. (11) can be expressed as

$$
\frac{\partial\left[\boldsymbol{G}\left(\lambda_{j}\right)\right]}{\partial p}=\left.\frac{\partial \lambda_{j}}{\partial p} \frac{\partial \boldsymbol{G}(s)}{\partial s}\right|_{s=\lambda_{j}}+\left.\frac{\partial \boldsymbol{G}(s)}{\partial p}\right|_{s=\lambda_{j}}
$$

When Eq. (11) is premultiplied by $\boldsymbol{u}_{j}^{T}$, and the symmetry property of the system matrices is used, it may be observed that the second term of the equation vanishes due to Eq. (4). Substituting Eq. (12) into Eq. (11), we obtain

$$
\begin{aligned}
\boldsymbol{u}_{j}^{T}\left[\lambda_{j}^{2} \frac{\partial \boldsymbol{M}}{\partial p}+\left.\lambda_{j} \frac{\partial \boldsymbol{G}(s)}{\partial p}\right|_{s=\lambda_{j}}+\frac{\partial \boldsymbol{K}}{\partial p}\right] \boldsymbol{u}_{j} \\
\quad+\boldsymbol{u}_{j}^{T}\left[2 \lambda_{j} \frac{\partial \lambda_{j}}{\partial p} \boldsymbol{M}+\frac{\partial \lambda_{j}}{\partial p} \boldsymbol{G}\left(\lambda_{j}\right)+\left.\lambda_{j} \frac{\partial \lambda_{j}}{\partial p} \frac{\partial \boldsymbol{G}(s)}{\partial s}\right|_{s=\lambda_{j}}\right] \boldsymbol{u}_{j}=0
\end{aligned}
$$

Rearranging the preceding equation, the derivative of eigenvalues can be obtained as

$$
\begin{gathered}
\frac{\partial \lambda_{j}}{\partial p}=-\left\{\boldsymbol{u}_{j}^{T}\left[\lambda_{j}^{2} \frac{\partial \boldsymbol{M}}{\partial p}+\left.\lambda_{j} \frac{\partial \boldsymbol{G}(s)}{\partial p}\right|_{s=\lambda_{j}}+\frac{\partial \boldsymbol{K}}{\partial p}\right] \boldsymbol{u}_{j} /\right. \\
\left.\boldsymbol{u}_{j}^{T}\left[2 \lambda_{j} \boldsymbol{M}+\boldsymbol{G}\left(\lambda_{j}\right)+\left.\lambda_{j} \frac{\partial \boldsymbol{G}(s)}{\partial s}\right|_{s=\lambda_{j}}\right] \boldsymbol{u}_{j}\right\}
\end{gathered}
$$

Note that the denominator of Eq. (14) is exactly the normalization relationship given by Eq. (10). In view of this, Eq. (14) can be expressed in a concise form as

$$
\frac{\partial \lambda_{j}}{\partial p}=-\left[\left.\boldsymbol{u}_{j}^{T} \frac{\partial \boldsymbol{D}(s)}{\partial p}\right|_{s=\lambda_{j}} \boldsymbol{u}_{j} /\left.\boldsymbol{u}_{j}^{T} \frac{\partial \boldsymbol{D}(s)}{\partial s}\right|_{s=\lambda_{j}} \boldsymbol{u}_{j}\right]
$$

or

$$
\frac{\partial \lambda_{j}}{\partial p}=-\frac{1}{\theta_{j}}\left(\left.\boldsymbol{u}_{j}^{T} \frac{\partial \boldsymbol{D}(s)}{\partial p}\right|_{s=\lambda_{j}} \boldsymbol{u}_{j}\right)
$$

This is the most general expression for the derivative of eigenvalues of linear dynamic systems. Equation (15) can be used to derive the derivative of eigenvalues for various special cases.

1) For the undamped systems ${ }^{1} \boldsymbol{G}(s)=0$ results,

$$
\boldsymbol{D}(s)=s^{2} \boldsymbol{M}+\boldsymbol{K}, \quad \theta_{j}=2 \lambda_{j} \boldsymbol{u}_{j}^{T} \boldsymbol{M} \boldsymbol{u}_{j}
$$

Assuming $\lambda_{j}=i \omega_{j}$, from Eq. (15), one obtains

$$
-2 i \omega_{j} i \frac{\partial \omega_{j}}{\partial p}=\frac{\partial \omega_{j}^{2}}{\partial p}=\left\{\boldsymbol{u}_{j}^{T}\left[\frac{\partial \boldsymbol{K}}{\partial p}-\omega_{j}^{2} \frac{\partial \boldsymbol{M}}{\partial p}\right] \boldsymbol{u}_{j} / \boldsymbol{u}_{j}^{T} \boldsymbol{M} \boldsymbol{u}_{j}\right\}
$$

which is a well known result.
2) For the viscously damped systems ${ }^{10,15} \boldsymbol{G}(s)=\boldsymbol{C}$, a constant matrix with respect to $s$. This results in

$$
\boldsymbol{D}(s)=s^{2} \boldsymbol{M}+s \boldsymbol{C}+\boldsymbol{K}, \quad \theta_{j}=\boldsymbol{u}_{j}^{T}\left[2 \lambda_{j} \boldsymbol{M}+\boldsymbol{C}\right] \boldsymbol{u}_{j}
$$

Using these, from Eq. (15), one obtains

$$
\frac{\partial \lambda_{j}}{\partial p}=-\left\{\boldsymbol{u}_{j}^{T}\left[\lambda_{j}^{2} \frac{\partial \boldsymbol{M}}{\partial p}+\lambda_{j} \frac{\partial \boldsymbol{C}}{\partial p}+\frac{\partial \boldsymbol{K}}{\partial p}\right] \boldsymbol{u}_{j} / \boldsymbol{u}_{j}^{T}\left[2 \lambda_{j} \boldsymbol{M}+\boldsymbol{C}\right] \boldsymbol{u}_{j}\right\}
$$

Thus, the result obtained in Eq. (15) generalize earlier expressions of the derivative of eigenvalues. A further generalization, when the system matrices are not symmetric is reported in Appendix A. The derivatives of associated eigenvectors are considered in the next section.

\section{Derivative of Eigenvectors}

The various methods of calculating the derivative of eigenvectors can be divided into three main categories ${ }^{7}: 1$ ) adjoint method or modal method, 2) direct method, and 3) iterative method. In this paper we adopt the modal method, where the derivative of each eigenvector is expanded in the space of the complete set of eigenvectors. The main difficulty in applying available methodologies for the modal method to nonviscously damped systems is that the eigenvectors do not satisfy any familiar orthogonality relationship. We propose a new approach to calculate the derivatives of eigenvectors without using the orthogonality relationship.

It turns out that the eigenvalue problem of the dynamic stiffness matrix [given by Eq. (3)] plays an important role. For any given $s \in \mathbb{C}$, the eigenvalue problem associated with the dynamic stiffness matrix can be expressed by

$$
\boldsymbol{D}(s) \phi_{k}(s)=v_{k}(s) \phi_{k}(s), \quad \forall k=1, \ldots, N
$$

In the Eq. (20), the eigenvalues $v_{k}(s) \in \mathbb{C}$ are the roots of the characteristic equation

$$
\operatorname{det}[\boldsymbol{D}(s)-v(s) \boldsymbol{I}]=0
$$

and $\phi_{k}(s) \in \mathbb{C}^{N}$ is the $k$ th eigenvector of $\boldsymbol{D}(s)$. It is assumed that all of the eigenvalues are distinct for any fixed value of $s$. The symbols $v_{k}(s)$ and $\phi_{k}(s)$ indicate functional dependence of these quantities on the complex parameter $s$. Such a continuous dependence is expected whenever $\boldsymbol{D}(s)$ is a sufficiently smooth matrix function of $s$. Note that, because $\boldsymbol{D}(s)$ is an $N \times N$ complex matrix for a fixed $s$, the number of eigenvalues (and consequently the eigenvectors) must be $N$. Furthermore, it can be shown that, for distinct eigenvalues, $\phi_{k}(s)$ also satisfy an orthogonality relationship, although $\boldsymbol{u}_{k}$ do not enjoy any such simple relationship. We normalize $\phi_{k}(s)$ such that

$$
\phi_{j}^{T}(s) \phi_{k}(s)=\delta_{k j}, \quad \forall k, j=1, \ldots, N
$$

In view of the preceding relationship, from Eq. (20) we have

$$
\phi_{j}^{T}(s) \boldsymbol{D}(s) \phi_{k}(s)=v_{k}(s) \delta_{k j}, \quad \forall k, j=1, \ldots, N
$$

or in matrix form,

$$
\Phi^{T}(s) \boldsymbol{D}(s) \Phi(s)=\nu(s)
$$

Here

$$
\begin{gathered}
\Phi(s)=\left[\phi_{1}(s), \phi_{2}(s), \ldots, \phi_{N}(s)\right] \in \mathbb{C}^{N \times N} \\
\nu(s)=\operatorname{diag}\left[v_{1}(s), v_{2}(s), \ldots, v_{N}(s)\right] \in \mathbb{C}^{N \times N}
\end{gathered}
$$

It is possible to establish the relationships between the original eigenvalue problem of the system defined by Eq. (4) and that by Eq. (20). Consider the case when the parameter $s$ approaches any one of the system eigenvalues, for example, $\lambda_{j}$. Because all of the $v_{k}(s)$ are assumed to be distinct, for nontrivial eigenvectors, by comparing Eqs. (4) and (20) we can conclude that one and only one of the $v_{k}(s)$ must be zero ${ }^{31}$ when $s \rightarrow \lambda_{j}$. Suppose that the $r$ th eigenvalue of the eigenvalue problem (20) is zero when $s \rightarrow \lambda_{j}$. It is also clear that the eigenvector in Eq. (20) corresponding to the $r$ th eigenvalue 
also approaches the eigenvector in Eq. (4) as $s \rightarrow \lambda_{j}$. Thus, when $s=\lambda_{j}$, one has

$v_{r}\left(\lambda_{j}\right)=0, \quad v_{k}\left(\lambda_{j}\right) \neq 0, \quad \forall k=1, \ldots, N ; \quad \neq r$

and also

$$
\phi_{r}\left(\lambda_{j}\right)=\boldsymbol{u}_{j}
$$

These equations completely relate the eigensolutions of Eq. (4) with Eq. (20). Now, these relationships will be utilized to obtain the derivative of eigenvectors of system (4).

When the $r$ th set is considered, Eq. (20) can be rewritten as

$$
Z_{r}(s) \phi_{r}(s)=\mathbf{0}
$$

where

$$
\boldsymbol{Z}_{r}(s)=\boldsymbol{D}(s)-v_{r}(s) \boldsymbol{I} \in \mathbb{C}^{N \times N}
$$

In view of Eq. (27), from Eq. (30) it is clear that

$$
\lim _{s \rightarrow \lambda_{j}} \boldsymbol{Z}_{r}(s)=\left.\boldsymbol{D}(s)\right|_{s=\lambda_{j}}
$$

From Eq. (31) together with Eq. (28), we conclude that in the limit $s \rightarrow \lambda_{j}$ the eigenvalue problem given by Eq. (29) approaches the original eigenvalue problem given by Eq. (4).

Differentiating Eq. (29) with respect to the design parameter $p$, one has

$$
\begin{gathered}
\frac{\partial \boldsymbol{Z}_{r}(s)}{\partial p} \phi_{r}(s)+\boldsymbol{Z}_{r}(s) \frac{\partial \phi_{r}(s)}{\partial p}=\mathbf{0} \text { or } \\
\boldsymbol{Z}_{r}(s) \frac{\partial \phi_{r}(s)}{\partial p}=-\frac{\partial \boldsymbol{Z}_{r}(s)}{\partial p} \phi_{r}(s)
\end{gathered}
$$

Premultiplying Eq. (32) by $\boldsymbol{D}^{-1}(s)$ and using Eq. (30), we have

$$
\left[\boldsymbol{I}-\boldsymbol{D}^{-1}(s) v_{r}(s)\right] \frac{\partial \phi_{r}(s)}{\partial p}=-\boldsymbol{D}^{-1}(s) \frac{\partial \boldsymbol{Z}_{r}(s)}{\partial p} \phi_{r}(s)
$$

The derivative of eigenvector of the original system with respect to the design parameter $p$, that is, $\partial \boldsymbol{u}_{j} / \partial p$, should be obtained from Eq. (33) by taking the limit $s \rightarrow \lambda_{j}$. Because $\lim _{s \rightarrow \lambda_{j}} \boldsymbol{D}(s)$ is at most of rank $(N-1)$, it is not possible to obtain $\partial \boldsymbol{u}_{j} / \partial p$ directly from Eq. (33). We avoid this difficulty by expanding $\boldsymbol{D}^{-1}(s)$ in terms of the poles and their associated residues.

From the definition of the matrix inverse, it is known that

$$
\boldsymbol{D}^{-1}(s)=\frac{\operatorname{adj}[\boldsymbol{D}(s)]}{\operatorname{det}[\boldsymbol{D}(s)]}
$$

Note that the poles of $\boldsymbol{D}^{-1}(s)$ are exactly the eigenvalues of the system as given by Eq. (5). Because it is assumed that all of the $m$ eigenvalues are distinct, each pole is a simple pole. Thus, $\boldsymbol{D}^{-1}(s)$ may be expressed in a pole-residue form as

$$
\boldsymbol{D}^{-1}(s)=\sum_{j=1}^{m} \frac{\boldsymbol{R}_{j}}{s-\lambda_{j}}
$$

where

$$
\boldsymbol{R}_{j}=\underset{s=\lambda_{j}}{\operatorname{res}}\left[\boldsymbol{D}^{-1}(s)\right] \stackrel{\text { def }}{=} \lim _{s \rightarrow \lambda_{j}}\left(s-\lambda_{j}\right)\left[\boldsymbol{D}^{-1}(s)\right]
$$

is the residue of $\boldsymbol{D}^{-1}(s)$ at the pole $\lambda_{j}$. Taking the inverse of Eq. (24) and rearranging, one can express $\boldsymbol{D}^{-1}(s)$ as

$$
\boldsymbol{D}^{-1}(s)=\boldsymbol{\Phi}(s) \boldsymbol{\nu}^{-1}(s) \boldsymbol{\Phi}^{T}(s)=\sum_{k=1}^{N} \frac{\phi_{k}(s) \phi_{k}^{T}(s)}{v_{k}(s)}
$$

When the $r$ th term of the right-hand side is separated, and Eq. (36) is used, the residue at $s=\lambda_{j}$ may be obtained:

$$
\begin{aligned}
\boldsymbol{R}_{j} \stackrel{\text { def }}{=} \lim _{s \rightarrow \lambda_{j}}\left(s-\lambda_{j}\right)\left\{\frac{\phi_{r}(s) \phi_{r}^{T}(s)}{v_{r}(s)}+\left[\sum_{\substack{k=1 \\
k \neq r}}^{N} \frac{\phi_{k}(s) \phi_{k}^{T}(s)}{v_{k}(s)}\right]\right\} \\
=\lim _{s \rightarrow \lambda_{j}}\left(s-\lambda_{j}\right) \frac{\phi_{r}(s) \phi_{r}^{T}(s)}{v_{r}(s)} \\
=\left[\left.\phi_{r}(s) \phi_{r}^{T}(s)\right|_{s=\lambda_{j}} /\left.\frac{\partial v_{r}(s)}{\partial s}\right|_{s=\lambda_{j}}\right]
\end{aligned}
$$$$
\text { (using l'Hôspital's rule) }
$$

$$
=\left[\boldsymbol{u}_{j} \boldsymbol{u}_{j}^{T} /\left.\frac{\partial v_{r}(s)}{\partial s}\right|_{s=\lambda_{j}}\right] \quad \text { [by Eq. (28)] }
$$

The denominator in the expression (38) for the residues $\partial v_{r}(s) /\left.\partial s\right|_{s=\lambda_{j}}$ can be determined as follows. Differentiate Eq. (29) with respect to $s$ to obtain

$$
\frac{\partial \boldsymbol{Z}_{r}(s)}{\partial s} \phi_{r}(s)+\boldsymbol{Z}_{r}(s) \frac{\partial \phi_{r}(s)}{\partial s}=\mathbf{0}
$$

Premultiplying Eq. (39) by $\phi_{r}^{T}(s)$, one obtains

$$
\phi_{r}^{T}(s) \frac{\partial \boldsymbol{Z}_{r}(s)}{\partial s} \phi_{r}(s)+\phi_{r}^{T}(s) \boldsymbol{Z}_{r}(s) \frac{\partial \phi_{r}(s)}{\partial s}=0
$$

When the transpose of Eq. (29) is taken and the symmetry property of $\boldsymbol{Z}_{r}(s)$ is considered, it follows that the second term of the left-hand side of the preceding equation is zero. When the normalizing condition in Eq. (22) is used, and the expression of $\boldsymbol{Z}_{r}(s)$ is substituted from Eq. (30), Eq. (40) reduces to

$$
\boldsymbol{\phi}_{r}^{T}(s) \frac{\partial \boldsymbol{D}(s)}{\partial s} \boldsymbol{\phi}_{r}(s)=\frac{\partial v_{r}(s)}{\partial s}
$$

With the limit $s \rightarrow \lambda_{j}$ taken, and Eq. (28) used, Eq. (41) yields

$$
\left.\frac{\partial v_{r}(s)}{\partial s}\right|_{s=\lambda_{j}}=\left.\boldsymbol{u}_{j}^{T} \frac{\partial \boldsymbol{D}(s)}{\partial s}\right|_{s=\lambda_{j}} \boldsymbol{u}_{j}
$$

Interestingly, observe that Eq. (42) is exactly the same as the normalization condition given by Eq. (10). In view of Eq. (42), Eq. (38) reads

$$
\boldsymbol{R}_{j}=\boldsymbol{u}_{j} \boldsymbol{u}_{j}^{T} / \theta_{j}
$$

Substituting $\boldsymbol{D}^{-1}(s)$ from Eq. (35) into Eq. (33), using Eqs. (27) and (43), and taking the limit as $s \rightarrow \lambda_{j}$, one obtains

$$
\begin{aligned}
\frac{\partial \boldsymbol{u}_{j}}{\partial p} & =-\lim _{s \rightarrow \lambda_{j}} \sum_{k=1}^{m} \frac{\boldsymbol{u}_{k} \boldsymbol{u}_{k}^{T}}{\theta_{j}\left(s-\lambda_{k}\right)} \frac{\partial \boldsymbol{Z}_{r}(s)}{\partial p} \phi_{r}(s) \\
& =a_{j j} \boldsymbol{u}_{j}-\sum_{\substack{k=1 \\
k \neq j}}^{m}\left[\left.\boldsymbol{u}_{k}^{T} \frac{\partial \boldsymbol{D}(s)}{\partial p}\right|_{s=\lambda_{j}} \boldsymbol{u}_{j} / \theta_{k}\left(\lambda_{j}-\lambda_{k}\right)\right] \boldsymbol{u}_{k}
\end{aligned}
$$

where

$$
a_{j j}=-\lim _{s \rightarrow \lambda_{j}}\left[\boldsymbol{u}_{j}^{T} \frac{\partial \boldsymbol{Z}_{r}(s)}{\partial p} \boldsymbol{\phi}_{r}(s) / \theta_{j}\left(s-\lambda_{j}\right)\right]
$$

In deriving Eq. (44), we have also made use of the relationships (28) and (31). Note that the limiting value of $a_{j j}$, the coefficient associated with $\boldsymbol{u}_{j}$, cannot be obtained from Eq. (45) because the denominator approaches to zero in the limit. In Appendix B, a different approach is presented to bypass this difficulty. 
From Eq. (B11) of Appendix B, one obtains

$$
a_{j j}=-\left\{\left.\boldsymbol{u}_{j}^{T} \frac{\partial^{2}[\boldsymbol{D}(s)]}{\partial s \partial p}\right|_{s=\lambda_{j}} \boldsymbol{u}_{j} / 2\left[\left.\boldsymbol{u}_{j}^{T} \frac{\partial \boldsymbol{D}(s)}{\partial s}\right|_{s=\lambda_{j}} \boldsymbol{u}_{j}\right]\right\}
$$

The denominator in Eq. (46) can be related to the normalization constant $\theta_{j}$ given by Eq. (10). The term

$$
\left.\frac{\partial^{2}[\boldsymbol{D}(s)]}{\partial s \partial p}\right|_{s=\lambda_{j}}
$$

appearing in the numerator may be obtained by differentiating Eq. (3) as

$$
\left.\frac{\partial^{2}[\boldsymbol{D}(s)]}{\partial s \partial p}\right|_{s=\lambda_{j}}=2 \lambda_{j} \frac{\partial \boldsymbol{M}}{\partial p}+\left.\frac{\partial \boldsymbol{G}(s)}{\partial p}\right|_{s=\lambda_{j}}+\left.\lambda_{j} \frac{\partial^{2}[\boldsymbol{G}(s)]}{\partial s \partial p}\right|_{s=\lambda_{j}}
$$

From Eqs. (44) and (47), the derivative of $\boldsymbol{u}_{j}$ is obtained as

$$
\begin{aligned}
\frac{\partial \boldsymbol{u}_{j}}{\partial p} & =-\frac{1}{2 \theta_{j}}\left(\left.\boldsymbol{u}_{j}^{T} \frac{\partial^{2}[\boldsymbol{D}(s)]}{\partial s \partial p}\right|_{s=\lambda_{j}} \boldsymbol{u}_{j}\right) \boldsymbol{u}_{j} \\
& -\sum_{\substack{k=1 \\
k \neq j}}^{m}\left[\left.\boldsymbol{u}_{k}^{T} \frac{\partial \boldsymbol{D}(s)}{\partial p}\right|_{s=\lambda_{j}} \boldsymbol{u}_{j} / \theta_{k}\left(\lambda_{j}-\lambda_{k}\right)\right] \boldsymbol{u}_{k}
\end{aligned}
$$

This is the most general expression for the derivative of eigenvectors of linear dynamic systems. Equation (48) can be applied directly to derive the derivative of eigenvectors for various special cases.

1) For the undamped systems ${ }^{1} \boldsymbol{G}(s)=0$ results, the order of the characteristic polynomial $m=2 N ; \lambda_{j}$ is purely imaginary so that $\lambda_{j}=i \omega_{j}$. By use of Eq. (16), Eq. (47) results in

$$
\left.\frac{\partial^{2}[\boldsymbol{D}(s)]}{\partial s \partial p}\right|_{s=\lambda_{j}}=2 \lambda_{j} \frac{\partial \boldsymbol{M}}{\partial p}
$$

Recalling that the eigenvalues appear in complex conjugate pairs and all $\boldsymbol{u}_{j}$ are real, from (48) one obtains

$$
\begin{aligned}
\frac{\partial \boldsymbol{u}_{j}}{\partial p} & =-\frac{1}{2}\left[\left(2 i \omega_{j} \boldsymbol{u}_{j}^{T} \frac{\partial \boldsymbol{M}}{\partial p} \boldsymbol{u}_{j}\right) / 2 i \omega_{j}\left(\boldsymbol{u}_{j}^{T} \boldsymbol{M} \boldsymbol{u}_{j}\right)\right] \boldsymbol{u}_{j} \\
& -\sum_{\substack{k=1 \\
k \neq j}}^{N}\left\{\boldsymbol{u}_{k}^{T}\left[\frac{\partial \boldsymbol{K}}{\partial p}-\omega_{j}^{2} \frac{\partial \boldsymbol{M}}{\partial p}\right] \boldsymbol{u}_{j} / 2 i \omega_{k}\left(\boldsymbol{u}_{k}^{T} \boldsymbol{M} \boldsymbol{u}_{k}\right)\right\} \\
& \times\left[\frac{1}{i \omega_{j}-i \omega_{k}}-\frac{1}{i \omega_{j}+i \omega_{k}}\right] \boldsymbol{u}_{k}
\end{aligned}
$$

Consider the unity mass normalization, that is, $\boldsymbol{u}_{k}^{T} \boldsymbol{M} \boldsymbol{u}_{k}=1$, $\forall k=1, \ldots, N$; Eq. (50) can be rewritten as

$$
\begin{aligned}
\frac{\partial \boldsymbol{u}_{j}}{\partial p} & =-\frac{1}{2}\left(\boldsymbol{u}_{j}^{T} \frac{\partial \boldsymbol{M}}{\partial p} \boldsymbol{u}_{j}\right) \boldsymbol{u}_{j} \\
& +\sum_{\substack{k=1 \\
k \neq j}}^{N}\left\{\boldsymbol{u}_{k}^{T}\left[\frac{\partial \boldsymbol{K}}{\partial p}-\omega_{j}^{2} \frac{\partial \boldsymbol{M}}{\partial p}\right] \boldsymbol{u}_{j} /\left(\omega_{j}^{2}-\omega_{k}^{2}\right)\right\} \boldsymbol{u}_{k}
\end{aligned}
$$

which is a well known result.

2) For the viscously damped systems ${ }^{10,15} \boldsymbol{G}(s)=\boldsymbol{C}$, a constant matrix with respect to $s$ and $m=2 N$. When Eq. (18) is used, Eq. (47) results in

$$
\left.\frac{\partial^{2}[\boldsymbol{D}(s)]}{\partial s \partial p}\right|_{s=\lambda_{j}}=2 \lambda_{j} \frac{\partial \boldsymbol{M}}{\partial p}+\frac{\partial \boldsymbol{C}}{\partial p}
$$

Recalling that the eigenvalues and eigenvectors appear in complex conjugate pairs, from Eq. (48) one obtains

$$
\begin{aligned}
\frac{\partial \boldsymbol{u}_{j}}{\partial p} & =-\frac{1}{2 \theta_{j}}\left(\boldsymbol{u}_{j}^{T}\left[2 \lambda_{j} \frac{\partial \boldsymbol{M}}{\partial p}+\frac{\partial \boldsymbol{C}}{\partial p}\right] \boldsymbol{u}_{j}\right) \boldsymbol{u}_{j} \\
& -\frac{1}{\theta_{j}^{*} 2 i \Im\left(\lambda_{j}\right)}\left(\left.\boldsymbol{u}_{j}^{*^{T}} \frac{\partial \boldsymbol{D}(s)}{\partial p}\right|_{s=\lambda_{j}} \boldsymbol{u}_{j}\right) \boldsymbol{u}_{j}^{*} \\
& -\sum_{\substack{k=1 \\
k \neq j}}^{N}\left\{\left[\left.\boldsymbol{u}_{k}^{T} \frac{\partial \boldsymbol{D}(s)}{\partial p}\right|_{s=\lambda_{j}} \boldsymbol{u}_{j} / \theta_{k}\left(\lambda_{j}-\lambda_{k}\right)\right] \boldsymbol{u}_{k}\right. \\
& \left.+\left[\left.\boldsymbol{u}_{k}^{*^{T}} \frac{\partial \boldsymbol{D}(s)}{\partial p}\right|_{s=\lambda_{j}} ^{\boldsymbol{u}_{j}} / \theta_{k}^{*}\left(\lambda_{j}-\lambda_{k}^{*}\right)\right] \boldsymbol{u}_{k}^{*}\right\}
\end{aligned}
$$

Thus, the result obtained in Eq. (48) generalizes earlier expressions of the derivative of eigenvectors. A further generalization, when the system matrices are asymmetric, is derived in Appendix A.

\section{Example}

We consider a two-degree-of-freedom system shown in Fig. 1 to illustrate a possible use of the expressions derived so far. The system considered here is similar to the one used by Adhikari, ${ }^{10}$ except that the dissipative element connected between the two masses is not a simple viscous dashpot but a nonviscous damper. The equations of motion describing the free vibration of the system can be expressed by Eq. (1), with

$$
\begin{gathered}
\boldsymbol{M}=\left[\begin{array}{ll}
m & 0 \\
0 & m
\end{array}\right], \quad \boldsymbol{K}=\left[\begin{array}{cc}
k_{1}+k_{3} & -k_{3} \\
-k_{3} & k_{2}+k_{3}
\end{array}\right] \\
G(t)=g(t) \hat{\boldsymbol{I}}
\end{gathered}
$$

where

$$
\hat{\boldsymbol{I}}=\left[\begin{array}{cc}
1 & -1 \\
-1 & 1
\end{array}\right]
$$

The damping function $g(t)$ is assumed to be the Golla-HughesMcTavish model (see Refs. 23 and 32) so that

$$
g(t)=c\left(\mu_{1} e^{-\mu_{1} t}+\mu_{2} e^{-\mu_{2} t}\right), \quad c, \mu_{1}, \mu_{2} \geq 0
$$

where $c$ is a constant and $\mu_{1}$ and $\mu_{2}$ are known as the relaxation parameters. In Eq. (56) if the function associated with $c$ were a delta function, $c$ would serve the purpose of the familiar viscous damping constant. Taking the Laplace transform of (55), one obtains

$$
\boldsymbol{G}(s)=G(s) \hat{\boldsymbol{I}}
$$

where

$$
G(s)=\mathcal{L}[g(t)]=c\left[\mu_{1} /\left(s+\mu_{1}\right)+\mu_{2} /\left(s+\mu_{2}\right)\right]
$$

When Eqs. (54) and (57) are substituted in Eq. (5), it may be shown that the system has six eigenvalues: four of which correspond to the two elastic modes (together with corresponding complex conjugate pairs) and the other two correspond to two nonviscous modes. For convenience, arrange the eigenvalues as

$$
\lambda_{e_{1}}, \quad \lambda_{e_{2}}, \quad \lambda_{e_{1}}^{*}, \quad \lambda_{e_{2}}^{*}, \quad \lambda_{n_{1}}, \quad \lambda_{n_{2}}
$$

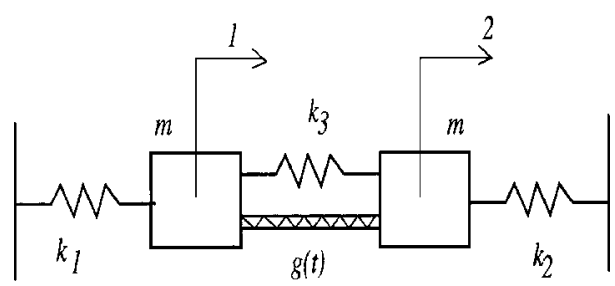

Fig. 1 Two-degree-of-freedom spring-mass system with nonviscous damping, $m=1 \mathrm{~kg}, k_{1}=1000 \mathrm{~N} / \mathrm{m}, k_{3}=100 \mathrm{~N} / \mathrm{m}, g(t)=c\left(\mu_{1} e^{-\mu_{1} t}+\right.$ $\left.\mu_{2} e^{-\mu_{2} t}\right), c=4.0 \mathrm{Ns} / \mathrm{m}, \mu_{1}=10.0 \mathrm{~s}^{-1}$, and $\mu_{2}=2.0 \mathrm{~s}^{-1}$. 
We consider the derivative of eigenvalues with respect to the relaxation parameter $\mu_{1}$. The derivative of the system matrices with respect to this parameter may be obtained as

$$
\frac{\partial \boldsymbol{M}}{\partial \mu_{1}}=\boldsymbol{O}, \quad \frac{\partial \boldsymbol{G}(s)}{\partial \mu_{1}}=\hat{\boldsymbol{I}} \frac{c s}{\left(s+\mu_{1}\right)^{2}}, \quad \frac{\partial \boldsymbol{K}}{\partial \mu_{1}}=\boldsymbol{O}
$$

Further, from Eq. (57), one also obtains

$$
\begin{gathered}
\frac{\partial \boldsymbol{G}(s)}{\partial s}=-\hat{\boldsymbol{I}} c\left\{\frac{\mu_{1}}{\left(s+\mu_{1}\right)^{2}}+\frac{\mu_{2}}{\left(s+\mu_{2}\right)^{2}}\right\} \\
\frac{\partial^{2}[\boldsymbol{G}(s)]}{\partial s \partial \mu_{1}}=-\hat{\boldsymbol{I}} c \frac{s-\mu_{1}}{\left(s+\mu_{1}\right)^{3}}
\end{gathered}
$$

When Eqs. (59) and (60) are used, the terms $\theta_{j}, \partial \boldsymbol{D}(s) / \partial p$, and $\partial^{2}[\boldsymbol{D}(s)] / \partial s \partial \mu_{1}$ appearing in Eqs. (15) and (48) can be evaluated.

Figures 2 and 3 show the real part of the derivative of first and second eigenvalue with respect to $\mu_{1}$ over a parameter variation of $k_{2}$ and $k_{3}$. These results are obtained by direct application of Eq. (15). The system considered here shows the so-called veering ${ }^{10}$ when the eigenvalues are plotted vs a system parameter. In the veering range, that is, when $k_{2} \approx k_{1}$ and $k_{3} \approx 0$, rapid changes take place in the eigensolutions. From Figs. 2 and 3 note that around the veering range the first eigenvalue is not very sensitive to $\mu_{1}$, whereas the second

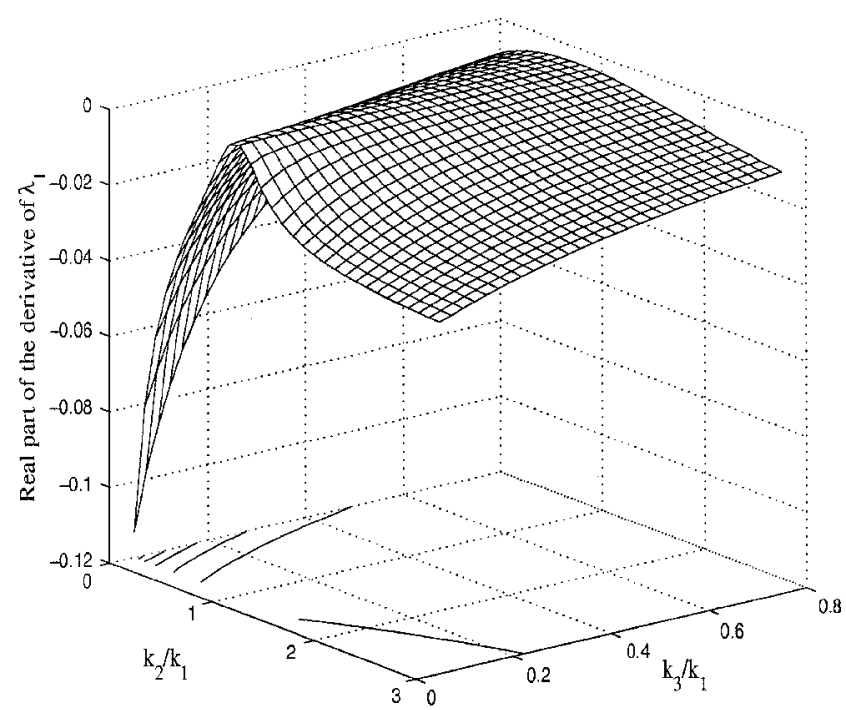

Fig. 2 Real part of the derivative of the first eigenvalue with respect to the relaxation parameter $\mu_{1}$.

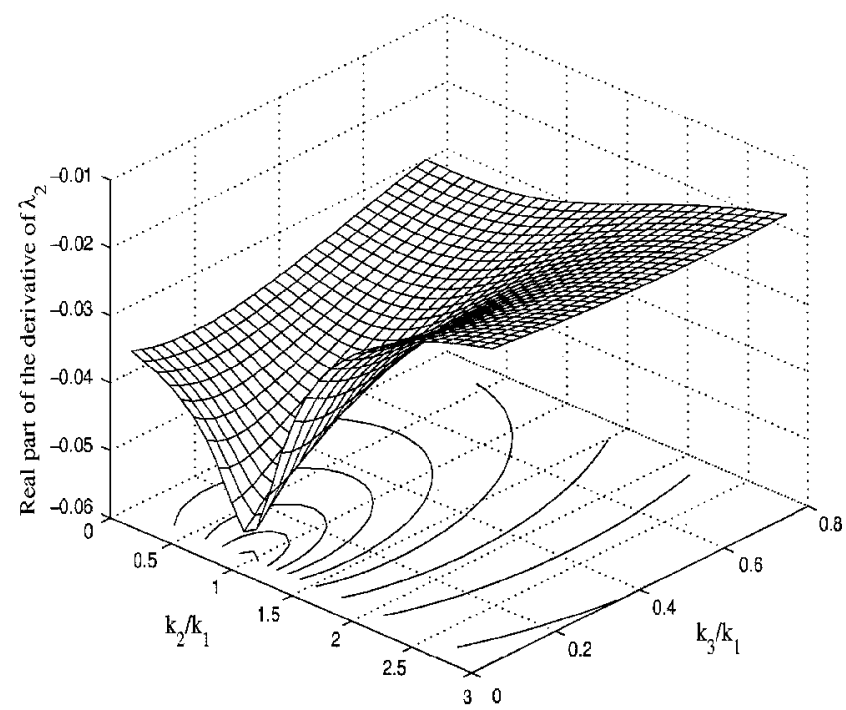

Fig. 3 Real part of the derivative of the second eigenvalue with respect to the relaxation parameter $\mu_{1}$.

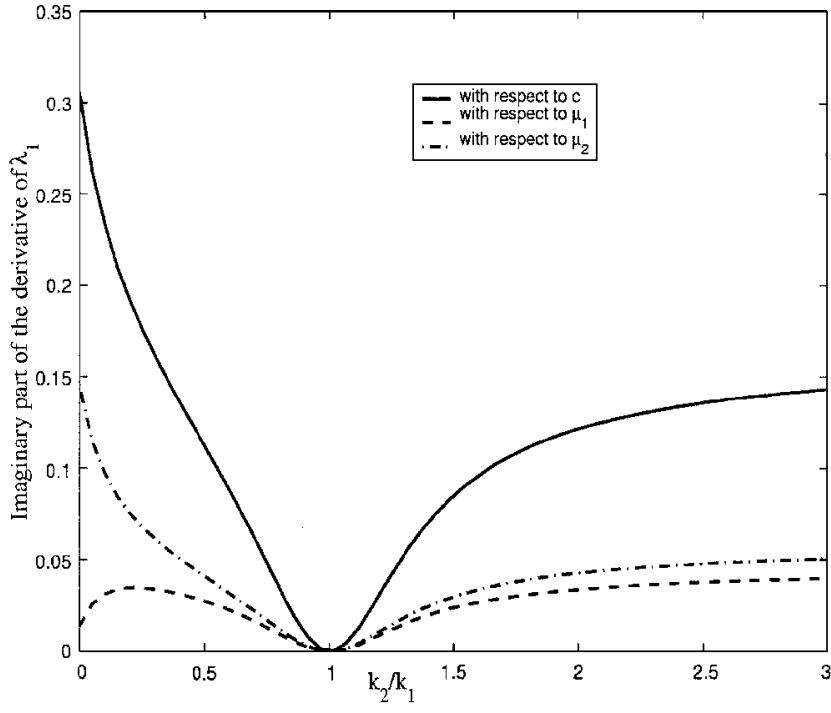

Fig. 4 Imaginary part of the derivative of the first eigenvalue with respect to the damping parameters $c, \mu_{1}$, and $\mu_{2}$.

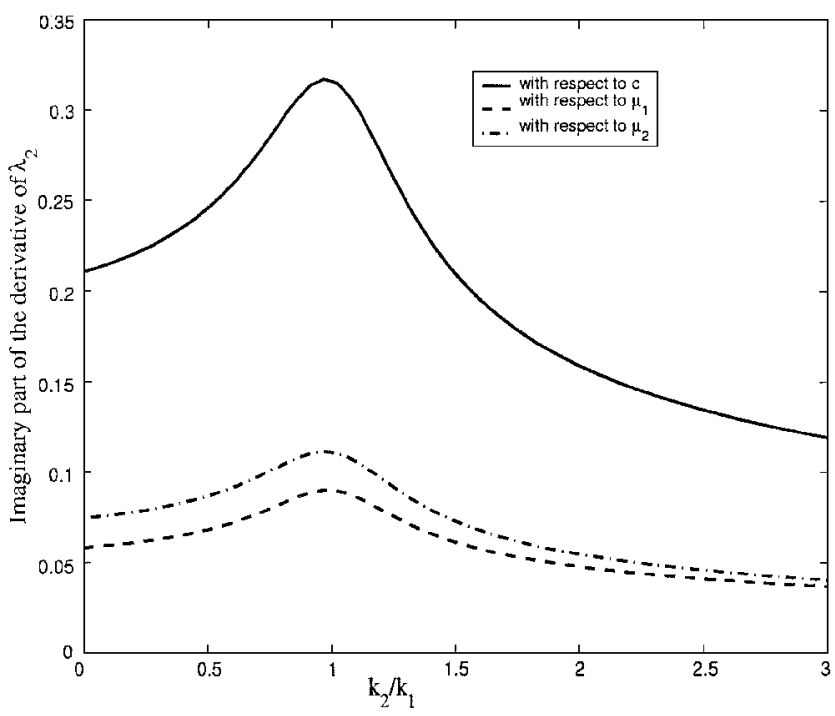

Fig. 5 Imaginary part of the derivative of the second eigenvalue with respect to the damping parameters $c, \mu_{1}$, and $\mu_{2}$.

eigenvalue is very sensitive in this region. In the first mode, both the blocks move in the same direction and consequently the damper is not stretched, resulting insensitivity to the relaxation parameter $\mu_{1}$. In the second mode the blocks move away from each other. This results stretching of the damping block and increases sensitivity to the relaxation parameter $\mu_{1}$.

It is useful to understand the effect of different parameters on the eigenvalues. Figures 4 and 5 show the imaginary part of the derivative of the first and second eigenvalues with respect to the damping parameters $c, \mu_{1}$, and $\mu_{2}$ over a parameter variation of $k_{2}$. The value of $k_{3}$ is fixed at $k_{3}=100$. Figures 4 and 5 show that the damping parameters not only affect the real part of the eigenvalues but also affect the imaginary part. Again, observe that in the veering range the first eigenvalue is insensitive to the damping parameters, whereas the second eigenvalue is sensitive to them.

Now we turn our attention to the derivative of eigenvectors. Figures 6 and 7 show the real part of the derivative of first and second eigenvectors with respect to $k_{2}$ over a parameter variation of $k_{2}$. It is useful to compare these results with the corresponding results by considering the damping mechanism to be viscous, that is, when $g(t)$ given by Eq. (56) has the form $g(t)=c \delta(t)$. In Figs. 6 and 7 , the derivative of both eigenvectors for the corresponding viscously damped system is also plotted. Observe that around the 


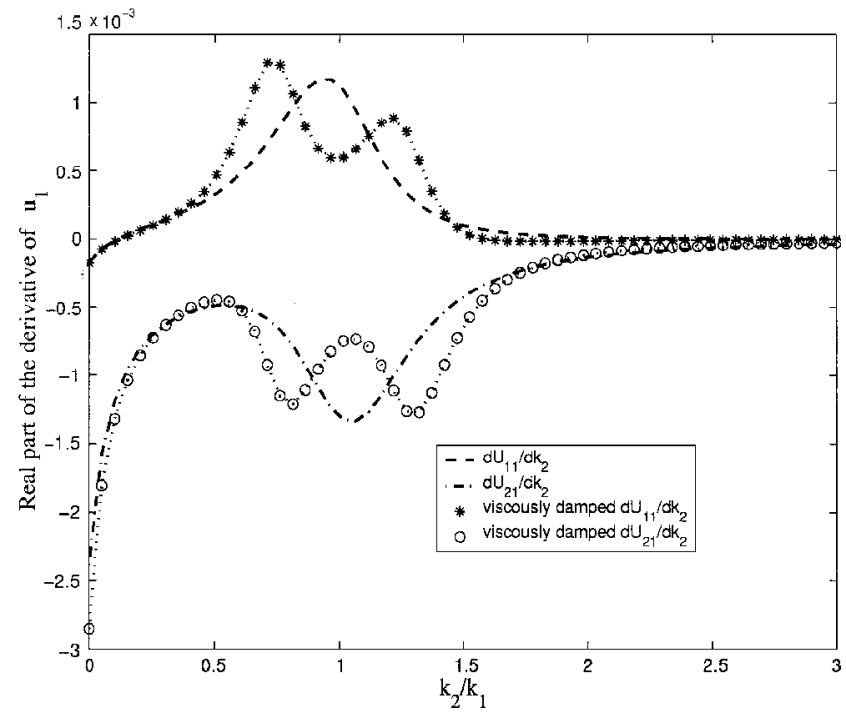

Fig. 6 Real part of the derivative of the first eigenvector with respect to $k_{2}$.

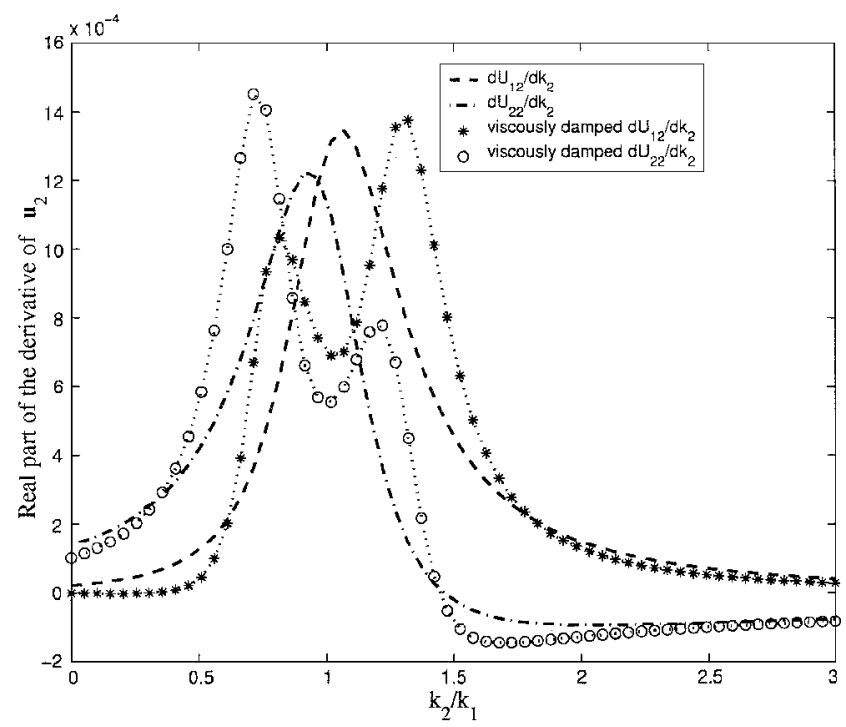

Fig. 7 Real part of the derivative of the second eigenvalue with respect to $k_{2}$.

veering range the derivatives of both eigenvectors are different for viscously and nonviscously damped systems. This illustrates that the nature of damping affects the parameter sensitivity of the real part of complex modes.

\section{Conclusions}

In general, structural systems are expected to be nonviscously damped. The derivatives of eigenvalues and eigenvectors of nonviscously damped discrete linear systems have been derived. The assumed nonviscous damping forces depend on the past history of velocities via convolution integrals over suitable kernel functions. The familiar viscous damping model is a special case corresponding to a memoryless kernel. It has been assumed that, in general, the mass and the stiffness matrices as well as the matrix of the kernel functions cannot be simultaneously diagonalized by any linear transformation. The analysis is, however, restricted to systems with nonrepetitive eigenvalues and nonsingular mass matrices.

Eigenvectors of linear dynamic systems with general nonviscous damping do not satisfy any kind of orthogonality relationship (not even in the usual state space). For this reason, none of the established methodologies for determination of the derivative of eigenvectors are applicable to nonviscously damped systems. In this paper, a new approach is developed that utilizes the eigenvalue problem of the associated complex dynamic stiffness matrix. The original eigenvalue problem is a limiting case of this eigenvalue problem. The expressions derived for the derivative of eigenvalues and eigenvectors [Eqs. (15) and (48)] are very general and also valid for undamped and viscously damped systems. The study conducted here opens up the possibility of extending the conventional modal updating and parameter estimation techniques to nonviscously damped systems, and further research in this direction is worth perusing.

\section{Appendix A: Asymmetric Systems}

In this section we consider a more general case when $\boldsymbol{M}, \boldsymbol{G}(t)$, and $\boldsymbol{K}$ are not restricted to symmetric matrices. For such asymmetric systems, the adjoint eigenvalue problem or left eigenvalue problem is defined as

$$
\boldsymbol{v}_{j}^{T}\left[\lambda_{j}^{2} \boldsymbol{M}+\lambda_{j} \boldsymbol{G}\left(\lambda_{j}\right)+\boldsymbol{K}\right]=\mathbf{0}^{T} \quad \text { or } \quad \boldsymbol{v}_{j}^{T} \boldsymbol{D}\left(\lambda_{j}\right)=\mathbf{0}^{T}
$$

where $\boldsymbol{v}_{j} \in \mathbb{C}^{N}$ is the $j$ th left eigenvector. Recently, Friswell and Adhikari $^{14}$ and Adhikari and Friswell ${ }^{15}$ derived the derivative of eigensolutions of viscously damped asymmetric linear systems. Following the approach developed by Adhikari and Friswell, ${ }^{15}$ it may be shown that the derivative of eigenvalues of nonviscously damped asymmetric systems is given by

$$
\frac{\partial \lambda_{j}}{\partial p}=-\frac{1}{\theta_{j}}\left[\left.\boldsymbol{v}_{j}^{T} \frac{\partial \boldsymbol{D}(s)}{\partial p}\right|_{s=\lambda_{j}} \boldsymbol{u}_{j}\right]
$$

where the normalization constants

$$
\theta_{j}=\left.\boldsymbol{v}_{j}^{T} \frac{\partial \boldsymbol{D}(s)}{\partial s}\right|_{s=\lambda_{j}} \boldsymbol{u}_{j}
$$

The method developed in this paper for determination of the derivative of eigenvectors can also be extended to asymmetric systems. The basic approach remains very much the same, except that suitable care in line with Ref. 15 must be taken to handle the asymmetry of the system matrices. The derivatives of right and left eigenvectors of nonviscously damped asymmetric systems are given by

$$
\begin{gathered}
\frac{\partial \boldsymbol{u}_{j}}{\partial p}=a_{j j} \boldsymbol{u}_{j}-\sum_{\substack{k=1 \\
k \neq j}}^{m}\left[\left.\boldsymbol{v}_{k}^{T} \frac{\partial \boldsymbol{D}(s)}{\partial p}\right|_{s=\lambda_{j}} \boldsymbol{u}_{j} / \theta_{k}\left(\lambda_{j}-\lambda_{k}\right)\right] \boldsymbol{u}_{k} \\
\frac{\partial \boldsymbol{v}_{j}}{\partial p}=b_{j j} \boldsymbol{v}_{j}-\sum_{\substack{k=1 \\
k \neq j}}^{m}\left[\left.\boldsymbol{v}_{j}^{T} \frac{\partial \boldsymbol{D}(s)}{\partial p}\right|_{s=\lambda_{j}} \boldsymbol{u}_{k} / \theta_{k}\left(\lambda_{j}-\lambda_{k}\right)\right] \boldsymbol{v}_{k}
\end{gathered}
$$

where

$$
a_{j j}+b_{j j}=-\frac{1}{\theta_{j}}\left\{\left.\boldsymbol{v}_{j}^{T} \frac{\partial^{2}[\boldsymbol{D}(s)]}{\partial s \partial p}\right|_{s=\lambda_{j}} \boldsymbol{u}_{j}\right\}
$$

It is clear that, for symmetric systems $\boldsymbol{u}_{j}=\boldsymbol{v}_{j}$, Eq. (A2) for the derivatives of eigenvalues reduces to its corresponding case in Eq. (15) and also that the expressions for the derivatives of right and left eigenvectors given by Eqs. (A4) and (A5) reduce to their corresponding case in Eq. (48).

\section{Appendix B: Determination of $\boldsymbol{a}_{\boldsymbol{j}}$}

For a fixed value of $s, \phi_{k}(s), \forall k=1, \ldots, N$, form a complete basis. For this reason $\partial \phi_{r}(s) / \partial p \in \mathbb{C}^{N}$ can be expanded uniquely in terms of all $\phi_{k}(s)$, that is, one can write 


$$
\frac{\partial \phi_{r}(s)}{\partial p}=\sum_{k=1}^{N} \alpha_{k}^{(r)}(s) \phi_{k}(s)
$$

where $\alpha_{k}^{(r)}(s) \in \mathbb{C}$ are nonzero constants. The normalization relationship for the $r$ th mode can be expressed from Eq. (23) as

$$
\phi_{r}^{T}(s) \boldsymbol{D}(s) \phi_{r}(s)=v_{r}(s)
$$

Differentiating this equation with respect to the design parameter $p$, we obtain

$$
\begin{gathered}
\frac{\partial \phi_{r}^{T}(s)}{\partial p} \boldsymbol{D}(s) \phi_{r}(s)+\phi_{r}^{T}(s) \frac{\partial \boldsymbol{D}(s)}{\partial p} \phi_{r}(s) \\
+\phi_{r}^{T}(s) \boldsymbol{D}(s) \frac{\partial \phi_{r}(s)}{\partial p}=\frac{\partial v_{r}(s)}{\partial p}
\end{gathered}
$$

When the symmetry property of $\boldsymbol{D}(s)$ and Eq. (30) are used, the Eq. (B3) can be rearranged as

$$
2 \phi_{r}^{T}(s) \boldsymbol{D}(s) \frac{\partial \phi_{r}(s)}{\partial p}=-\phi_{r}^{T}(s) \frac{\partial \boldsymbol{Z}_{r}(s)}{\partial p} \phi_{r}(s)
$$

Substituting $\partial \phi_{r}(s) / \partial p$ from Eq. (B1) and using the orthogonality relationship given by Eq. (23) from the preceding equation, one obtains

$$
\alpha_{r}^{(r)}(s)=-\left[\phi_{r}^{T}(s) \frac{\partial \boldsymbol{Z}_{r}(s)}{\partial p} \phi_{r}(s) / 2 v_{r}(s)\right]
$$

Now, taking the limit $s \rightarrow \lambda_{j}$ on Eq. (B1) and using Eq. (28), we have

$$
\begin{gathered}
\lim _{s \rightarrow \lambda_{j}} \frac{\partial \phi_{r}(s)}{\partial p}=\lim _{s \rightarrow \lambda_{j}} \sum_{k=1}^{N} \alpha_{k}^{(r)}(s) \phi_{k}(s) \quad \text { or } \\
\frac{\partial \boldsymbol{u}_{j}}{\partial p}=\left[\lim _{s \rightarrow \lambda_{j}} \alpha_{r}^{(r)}(s)\right] \boldsymbol{u}_{j}+\lim _{s \rightarrow \lambda_{j}} \sum_{\substack{k=1 \\
k \neq r}}^{N} \alpha_{k}^{(r)}(s) \phi_{k}(s)
\end{gathered}
$$

Because it is assumed that all of the eigenvalues are distinct, the associated eigenvectors are also distinct. Thus, $\lim _{s \rightarrow \lambda_{j}} \phi_{k}(s) \neq \boldsymbol{u}_{j}$, $\forall k=1, \ldots, N ; \neq r$. Thus, when the coefficient of $\boldsymbol{u}_{j}$ in Eqs. (44) and (B6) are compared, it is clear that

$$
\begin{aligned}
a_{j j} & =\lim _{s \rightarrow \lambda_{j}} \alpha_{r}^{(r)}(s) \\
& =-\lim _{s \rightarrow \lambda_{j}}\left[\phi_{r}^{T}(s) \frac{\partial \boldsymbol{Z}_{r}(s)}{\partial p} \phi_{r}(s) / 2 v_{r}(s)\right]
\end{aligned}
$$

The preceding limit cannot be evaluated directly because from Eq. (27) $\lim _{s \rightarrow \lambda_{j}} v_{r}(s)=0$. Now, differentiate Eq. (29) with respect to $p$ to obtain

$$
\frac{\partial \boldsymbol{Z}_{r}(s)}{\partial p} \phi_{r}(s)+\boldsymbol{Z}_{r}(s) \frac{\partial \phi_{r}(s)}{\partial p}=\mathbf{0}
$$

Premultiplying Eq. (B8) by $\phi_{r}^{T}(s)$, one obtains

$$
\phi_{r}^{T}(s) \frac{\partial \boldsymbol{Z}_{r}(s)}{\partial p} \phi_{r}(s)+\phi_{r}^{T}(s) \boldsymbol{Z}_{r}(s) \frac{\partial \phi_{r}(s)}{\partial p}=0
$$

When the transpose of Eq. (29) is taken and the symmetry property of $\boldsymbol{Z}_{r}(s)$ is considered, it follows that the second term of the left-hand side of Eq. (B9) is zero. Thus, Eq. (B9) reduces to

$$
\phi_{r}^{T}(s) \frac{\partial \boldsymbol{Z}_{r}(s)}{\partial p} \phi_{r}(s)=0
$$

Equation (B10) shows that in the limit the left-hand side of Eq. (B7) has a 0 by 0 form. Thus, applying l'Hôspital's rule, using Eqs. (28), (31), and (42), from Eq. (B7), one obtains

$$
\begin{aligned}
a_{j j} & =-\left\{\left.\boldsymbol{u}_{j}^{T} \frac{\partial^{2}[\boldsymbol{D}(s)]}{\partial s \partial p}\right|_{s=\lambda_{j}} \boldsymbol{u}_{j} /\left.2 \frac{\partial v_{r}(s)}{\partial s}\right|_{s=\lambda_{j}}\right\} \\
& =-\left\{\left.\boldsymbol{u}_{j}^{T} \frac{\partial^{2}[\boldsymbol{D}(s)]}{\partial s \partial p}\right|_{s=\lambda_{j}} \boldsymbol{u}_{j} / 2\left(\left.\boldsymbol{u}_{j}^{T} \frac{\partial \boldsymbol{D}(s)}{\partial s}\right|_{s=\lambda_{j}} \boldsymbol{u}_{j}\right)\right\}
\end{aligned}
$$

This expression can now be used to obtain the derivative of $\boldsymbol{u}_{j}$ in Eq. (44).

\section{Acknowledgments}

Financial support provided by the Nehru Memorial Trust, London, and the Cambridge Commonwealth Trust is gratefully acknowledged.

\section{References}

${ }^{1}$ Fox, R. L., and Kapoor, M. P., "Rates of Change of Eigenvalues and Eigenvectors," AIAA Journal, Vol. 6, No. 12, 1968, pp. 2426-2429.

${ }^{2}$ Rogers, L. C., "Derivatives of Eigenvalues and Eigenvectors," AIAA Journal, Vol. 8, No. 5, 1970, pp. 943, 944.

${ }^{3}$ Plaut, R. H., and Huseyin, K., "Derivative of Eigenvalues and Eigenvectors in Non-Self-Adjoint Systems," AIAA Journal, Vol. 11, No. 2, 1973 pp. 250, 251.

${ }^{4}$ Garg, S., "Derivatives of Eigensolutions for a General Matrix," AIAA Journal, Vol. 11, No. 8, 1973, pp. 1191-1194.

${ }^{5}$ Rudisill, C. S., "Derivatives of Eigenvalues and Eigenvectors for a General Matrix,” AIAA Journal, Vol. 12, No. 5, 1974, pp. 721, 722.

${ }^{6}$ Nelson, R. B., "Simplified Calculation of Eigenvector Derivatives," AIAA Journal, Vol. 14, No. 9, 1976, pp. 1201-1205.

${ }^{7}$ Murthy, D. V., and Haftka, R. T., "Derivatives of Eigenvalue s and Eigenvectors of a General Complex Matrix," International Journal for Numerical Methods in Engineering, Vol. 26, No. 2, 1988, pp. 293-311.

${ }^{8}$ Zeng, Q. H., "Highly Accurate Modal Method for Calculating Eigenvector Derivatives in Viscous Damping Systems," AIAA Journal, Vol. 33 No. 4, 1995, pp. 746-751.

${ }^{9}$ Cardani, C., and Mantegazza, P., "Calculation of Eigenvalue and Eigenvector Derivatives for Algebraic Flutter and Divergence Eigenproblems," AIAA Journal, Vol. 17, No. 4, 1979, pp. 408-412.

${ }^{10}$ Adhikari, S., "Rates of Change of Eigenvalues and Eigenvectors in Damped Dynamic Systems," AIAA Journal, Vol. 37, No. 11, 1999, pp. 1452 1458 .

${ }^{11}$ Lee, I.-W., Kim, D.-O., and Jung, H.-H., "Natural Frequency and Mode Shape Sensitivities of Damped Systems: Part I, Distinct Natural Frequencies," Journal of Sound and Vibration, Vol. 223, No. 3, 1999, pp. 399-412.

${ }^{12}$ Lee, I.-W., Kim, D.-O., and Jung, H.-H., "Natural Frequency and Mode Shape Sensitivities of Damped Systems: Part II, Multiple Natural Frequencies," Journal of Sound and Vibration, Vol. 223, No. 3, 1999, pp. 413-424.

${ }^{13}$ Adhikari, S., "Calculation of Derivative of Complex Modes Using Classical Normal Modes," Computer and Structures, Vol. 77, No. 6, 2000, pp. 625-633.

${ }^{14}$ Friswell, M. I., and Adhikari, S., "Derivatives of Complex Eigenvector s Using Nelson's Method,” AIAA Journal, Vol. 38, No. 12, 2000, pp. 2355 2357.

${ }^{15}$ Adhikari, S., and Friswell, M. I., "Eigenderivative Analysis of Asymmetric Non-conservative Systems," International Journal for Numerical Methods in Engineering, Vol. 51, No. 6, 2001, pp. 709-733.

${ }^{16}$ Baburaj, V., and Matsukai, Y., "A Study on the Material Damping of Thin Angle-Ply Laminated Plates," Journal of Sound and Vibration, Vol. 172, No. 3, 1994, pp. 415-419.

${ }^{17}$ Earls, S., W. E., "Theoretical Estimation of Frictional Energy Dissipation in a Simple Lap Joint," Journal of Mechanical Engineering Science, Vol. 8, No. 2, 1966, pp. 207-214.

${ }^{18}$ Beards, C. F., and Williams, J. L., "The Damping of Structural Vibration by Rotational Slip in a Structural Joint," Journal of Sound and Vibration, Vol. 53, No. 3, 1977, pp. 333-340.

${ }^{19}$ Banks, H. T., and Inman, D. J., “On Damping Mechanisms in Beams," Journal of Applied Mechanics, Vol. 58, Sept. 1991, pp. 716-723.

${ }^{20}$ Adhikari, S., and Woodhouse, J., "Identification of Damping: Part 2, Non-viscous Damping," Journal of Sound and Vibration, Vol. 243, No. 1 , 2001, pp. 63-88.

${ }^{21}$ Adhikari, S., "Dynamics of Non-Viscously Damped Linear Systems,' Journal of Engineering Mechanics, Vol. 128, No. 3, 2002, pp. 328-339.

${ }^{22}$ Adhikari, S., "Classical Normal Modes in Nonviscousl y Damped Linear Systems,” AIAA Journal, Vol. 39, No. 5, 2001, pp. 978-980. 
${ }^{23}$ Golla, D. F., and Hughes, P. C., "Dynamics of Viscoelastic StructuresA Time Domain Finite Element Formulation," Journal of Applied Mechanics, Vol. 52, Dec. 1985, pp. 897-906.

${ }^{24}$ Bland, D. R., Theory of Linear Viscoelasticity, Pergamon, London, 1960.

${ }^{25}$ Muravyov, A., "Analytical Solutions in the Time Domain for Vibration Problems of Discrete Viscoelastic Systems," Journal of Sound and Vibration, Vol. 199, No. 2, 1997, pp. 337-348.

${ }^{26}$ Adhikari, S., "Eigenrelations for Nonviscously Damped Systems," AIAA Journal, Vol. 39, No. 8, 2001, pp. 1624-1630.

${ }^{27}$ Vigneron, F. R., "A Natural Modes Model and Modal Identities for Damped Linear Structures," Journal of Applied Mechanics, Vol. 53, March 1986, pp. 33-38.

${ }^{28}$ Sestieri, A., and Ibrahim, R., "Analysis of Errors and Approximation s in the Use of Modal Coordinates," Journal of Sound and Vibration, Vol. 177,
No. 2, 1994, pp. 145-157.

${ }^{29}$ Fawzy, I., "Orthogonality of Generally Normalized Eigenvectors and Eigenrows,” AIAA Journal, Vol. 15, No. 2, 1977, pp. 276-278.

${ }^{30}$ Adhikari, S., "Optimal Complex Modes and an Index of Damping Non-proportionality," Mechanical System and Signal Processing (to be published).

${ }^{31}$ Yang, B., and Wu, X., "Modal Expansion of Structural Systems with Time Delays," AIAA Journal, Vol. 36, No. 12, 1998, pp. 2218 2224.

${ }^{32}$ McTavish, D. J., and Hughes, P. C., "Modeling of Linear Viscoelastic Space Structures," Journal of Vibration and Acoustics, Vol. 115, Jan. 1993, pp. $103-110$.

C. Pierre 